\title{
Estudo sobre quedas na população geriátrica, da prevenção a morbimortalidade: uma revisão integrativa
}

\author{
Study on falls in the geriatric population, from prevention to morbimortality: an integrative \\ review
}
Estudio sobre caídas en población geriátrica, de la prevención a morbimortalidae: revisión integrativa

Matheus Hybner Gonçalves ${ }^{1 *}$, Jemima Ferreira Coelho', Girley Cordeiro de Sousa ${ }^{1}$.

\section{RESUMO}

Objetivo: Construir, por meio de um arcabouço teórico, um entendimento alicerçado e uniforme que reconheça a problemática em questão e que possa agregar conhecimento a prática clínica para população geriátrica. Métodos: Esse trabalho se trata de um estudo feito com base em uma revisão integrativa de literatura especializada e fundamentada em evidências. As etapas de elaboração foram: identificar o tema e selecionar a questão de pesquisa, buscar artigos em bases digitais e estabelecer critérios de inclusão e exclusão e análise com discussão dos resultados obtidos dos periódicos. Resultados: Pode inferir que os fatores de risco contribuem de forma significativa para a ocorrência da queda, e com ele suas complicações. Dessa forma, é imperativo a realização de uma avaliação ampla no idoso a fim de identificar os fatores de risco e prevenir a queda e seu desfecho. Considerações finais: Um atendimento multidisciplinar do paciente idoso é essencial, para que o médico possa ser capaz de identificar os fatores de risco para quedas presentes no processo de declínio fisiológico da vida. Buscando assim interver de maneira apropriada antes da ocorrência do evento. $O$ médico ainda deve ser capaz de estimular a manutenção da sua autonomia e independência para a realização das atividades diárias.

Palavras-chave: Pessoa idosa, Morbimortalidade, Fator de risco.

\begin{abstract}
Objective: To build, through a theoretical framework, a grounded and uniform understanding that recognizes the issue in question and that can add knowledge to clinical practice for the geriatric population. Methods: This work is a study based on an integrative review of specialized literature and based on evidence. The preparation steps were: identify the topic and select the research question, search for articles on digital bases and define inclusion and exclusion criteria and analysis with discussion of the results obtained from the journals. Results: It can be inferred that the risk factors contribute in a reduced way to the occurrence of the fall, and with it its complications. Thus, it is imperative to conduct a comprehensive assessment in the elderly in order to identify the risk factors and prevent falls and their outcome. Final considerations: Multidisciplinary care for elderly patients is essential, so that the doctor may be able to identify the risk factors for falls present in the process of physiological decline in life. Thus seeking to intervene in an appropriate manner before the event occurs. The doctor must still be able to encourage the maintenance of his autonomy and independence to carry out daily activities.
\end{abstract}

Key words: Elderly person, Morbidity and mortality, Risk factor.

${ }^{1}$ Universidade de Vassouras (UV), Vassouras - RJ. *E-mail: math_hybner@outlook.com 


\section{RESUMEN}

Objetivo: Construir, a través de un marco teórico, un entendimiento fundamentado y uniforme que reconozca el tema en cuestión y que pueda agregar conocimientos a la práctica clínica de la población geriátrica. Métodos: Este trabajo es un estudio basado en una revisión integradora de literatura especializada y basado en evidencia. Los pasos de preparación fueron: identificar el tema y seleccionar la pregunta de investigación, buscar artículos en bases digitales y definir criterios de inclusión y exclusión y análisis con discusión de los resultados obtenidos de las revistas. Resultados: Se puede inferir que los factores de riesgo contribuyen de forma reducida a la ocurrencia de la caída, y con ella sus complicaciones. Por tanto, es imperativo realizar una valoración integral en el anciano con el fin de identificar los factores de riesgo y prevenir las caídas y su evolución. Consideraciones finales: La atención multidisciplinar del paciente anciano es fundamental para que el médico pueda identificar los factores de riesgo de caídas presentes en el proceso de deterioro fisiológico de la vida. Buscando así intervenir de manera adecuada antes de que ocurra el evento. El médico aún debe poder fomentar el mantenimiento de su autonomía e independencia para realizar las actividades diarias.

Palabras clave: Anciano, Morbilidad y mortalidad, Factor de riesgo.

\section{INTRODUÇÃO}

No Brasil e no mundo está ocorrendo o processo de transição demográfica no qual há uma mudança da estratificação da pirâmide etária com diminuição da base e alargamento do topo onde se encontra a população geriátrica resultando um aumento da expectativa de vida e uma diminuição da taxa de fecundidade (LIMA MG, et al., 2016; SIQUEIRA GV, et al., 2007).

O indivíduo é considerado idoso a partir de 60 anos de idade, onde já é possível perceber o início do declínio das suas funções fisiológicas, biológicas e psicológicas o que acaba por tornar o indivíduo mais propenso ao adoecimento e consequentemente a morte (MESQUITA GV, et al., 2009; MOSQUÉRA JM e ALVES AR 2018).

Uma parte dos idosos acabam tendo uma perda da capacidade funcional além do esperado, e justamente por isso, estes indivíduos passaram a ser denominados de "idosos frágeis". Essa fragilidade é vista na forma de vários sinais e sintomas característicos, que uma vez reunidos passou a ser visto como síndrome do idoso frágil. Os sinais e sintomas que constituem essa síndrome são: fadiga, anorexia, osteopenia, sarcopenia, anormalidades no equilibro e marcha (FRIED LP, et al., 2001). Essas mudanças que ocorrem fisiologicamente com o envelhecimento do indivíduo podem quando associados a afecções agudas e/ou crônicas podem acarretar em limitações funcionais que torna a população idosa mais suscetível aos fatores ambientais aumentando assim a morbimortalidade dos paciente geriátrico (MESQUITA GV, et al., 2009; MOSQUÉRA JM e ALVES AR, 2018).

Um indivíduo hígido para ter sua estabilidade postural preservada depende de componentes sensoriais, cognitivos dos sistema nervoso central e integralidade musculoesquelética (RIBEIRO AP, et al., 2006). Dessa forma, se a pessoa que já estiver em declínio de suas condições fisiológicas for acometida por patologias ou afecções que comprometa qualquer um desses sistemas, esta pessoa se tornará mais propensa a ocorrência do evento da queda.

Sabe- se que as múltiplas etiologias que corroboram para a ocorrência do fenômeno da queda podem ser classificadas em fatores intrínsecos (alterações fisiológicas), está, por sua vez, se divide em: distúrbios de marcha, equilíbrio e acuidade visual. Por outro lado tem os fatores extrínsecos (ambiente), que se dividem em: iluminação inadequada, ausência de suportes corporais como corrimão e barras de apoio, inutilização de órteses, presença de pisos com baixa coeficiente de atrito ou decorações que impeçam esse coeficiente de atrito como os tapetes ou até mesmo o uso de calcados inadequados. No que tange a prevenção, é notório que, dentre os dois fatores, o fator extrínseco é o que se pode intervir com mais facilidade (CONFORTIN SC, et al., 2020; MESQUITA GV, et al., 2009; SIQUEIRA FV, et al., 2007; SMITH AA, et al., 2017). 
A grande preocupação do médico geriatra hoje com relação ao evento da queda, não é sua ocorrência em si e sim as consequências advindas com o trauma, dentre elas a mais temida é a fratura óssea e as complicações associadas a ela como a restrição ao leito, limitação funcional, dor crônica, osteomielite, síndrome comportamental, embolia gordurosa até mesmo ter a morte como desfecho (RIERA R, et al., 2003; STOLT LROG, et al., 2020). Diante do que foi exposto, este estudo teve como propósito apontar os principais fatores de riscos para o fenômeno de quedas, seus agravos e consequências.

\section{MÉTODOS}

Esse trabalho se trata de um estudo feito com base em uma revisão integrativa de literatura especializada, fundamentada em evidências, onde o objetivo é construir um entendimento alicerçado e uniforme que reconheça a problemática em questão e que possa agregar conhecimento uma prática clínica de qualidade. Ele se propõe a responder ao seguinte questionamento: "Quais são os principais fatores de risco a quedas e suas principais consequências?". Dessarte, as etapas de construção deste foram: identificar o tema e selecionar a questão de pesquisa, buscar artigos em bases digitais e estabelecer critérios de inclusão e exclusão com posterior análise e discussão dos resultados obtidos dos periódicos.

Foram buscados artigos publicados no período de 2000 a 2020, as bases de dados utilizadas foram Google Acadêmico, PubMed e Scielo. Utilizando os seguintes descritores: morbimortalidade, quedas e idosos, no Google Acadêmico e no Scielo em português e no PubMed em inglês e espanhol. No PubMed foram encontrados no total 281 artigos, filtrando os achados para o período de tempo 2002 a 2020, sendo encontrados 268 artigos, sendo selecionados os artigos de acordo com a relevância para o tema. Na base de dados Scielo as buscas resultaram em 10 artigos após seleção com filtro no período de tempo de 2010 a 2020 foi obtido 6 publicações. No Google acadêmico foram encontrados 5240 artigos, após a aplicação do filtro no período de 2002 a 2020 foi obtido 5020 .

De todos os arquivos encontrados nas três bases de dados pesquisadas foram excluídas as duplicatas, sendo assim utilizados apenas 22 publicações, com base nos critérios de inclusão: artigos baseados em evidências, consensos e diretrizes que tivessem como questão norteadora a prevenção, fatores de risco, morbidade e mortalidade do evento da queda em idoso, bem como suas possíveis repercussões para a qualidade de vida do paciente, foram excluídos artigos que tangenciam o tema ou que os descritores não estavam correlacionados a proposta de pesquisa conforme Figura 1 a seguir. Além disso, todas as referências dos 22 periódicos selecionados foram analisadas por completo a fim de ampliar o arcabouço teórico deste artigo.

Figura 1 - Fluxograma da seleção dos estudos para esta revisão.

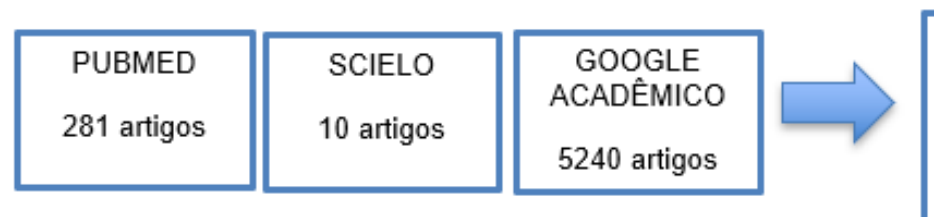

Critérios de exclusão: duplicação entre as bases e fora do tema abordado Critérios de inclusão: estudos disponíveis gratuitamente e que respondam a questão norteadora 23 artigos

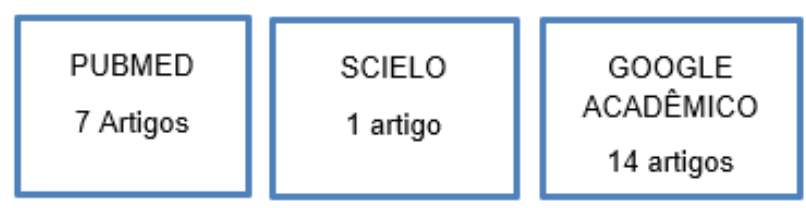

Fonte: Gonçalves MH, et al., 2021. 


\section{RESULTADOS}

No primeiro momento, com os descritores específicos foram selecionados por meio de filtros 23 artigos, que ao serem identificados como adequados ao propósito deste trabalho foram arquivados para uma leitura posterior e análise crítica. Posteriormente, ocorreu a leitura dos artigos selecionados passou-se a buscar acesso aos resumos de todos os exemplares, sendo cuidadosamente revisado em leituras disciplinares, avaliando os conteúdos pertinentes à temática.

Dos 23 artigos analisados podemos inferir que os fatores de risco são de suma importância para a identificação dos pacientes idosos que são mais suscetíveis ao evento da queda, sendo destes os principais fatores de risco são: idade avançada, sexo feminino e história prévia de quedas.

Outrossim, a sazonalidade também é um fator que deve ser levado em consideração na análise epidemiológica das internações e mortalidade de idosos que sofrem quedas. Foi visto que há um aumento no números de casos durante as estações mais frias como no outono e inverno devido às temperaturas mais baixas e chuvas intensas sobretudo no período diurno (STOLT LROG, et al., 2020). Ademais, os fatores extrínsecos são de igual importância aos fatores intrínsecos que podem ser prevenidos com higiene do ambiente para esse fim é importante que o profissional de saúde detenha a capacidade de reconhecer fatores de risco abordados no presente artigo para que as providências corretivas sejam tomadas o mais precoce possível, de forma a preservar a qualidade de vida do paciente envolvido.

Vale ressaltar que as os idosos apresentam maior risco de ocorrer fratura de fêmur e coluna vertebral. Dessa maneira, esse grupo etário apresenta maior necessidade acompanhamento ambulatorial e internações visto que as lesões decorrentes ao fenômeno de quedas podem requerer um longo período de tratamento aumentando as chances de complicações e desfavoráveis como cronicidade, incapacidade, invalidez e morte e como a maioria das fraturas são de caráter cirúrgico as complicações após o procedimento podem ser sérias como infecções, principalmente do trato urinário e na ferida operatória, pneumonia e delirium (MOSQUÉRA JM e ALVES AR, 2018)

Além disso, no que tange ao assunto de quedas, é interessante ressaltar a importância da integralidade, que é um dos princípios do Sistema Único de saúde (SUS), que visa garantir um completo do paciente, incluindo $o$ atendimento pré hospitalar e o serviços de emergência e urgência pois são porta de entrada para o atendimento desse idoso após o evento da queda, e também cuidados após a alta hospitalar. Isso pode ser feito com a participação de uma equipe multidisciplinar que inclui os serviços de fisioterapia, ortopedia, neurologia e geriatria seja no ambiente intra hospitalar para que o tempo de permanência diminuía com medidas como deambulação precoce e independência funcional precoce com uso seguro de auxiliares de marcha para que esse idoso diminui as complicações e o número de óbitos; seja no extra hospitalar com acompanhamento ambulatorial ou visitas domiciliares, onde é uma oportunidade de prevenir recorrência de outros episódios semelhantes e complicações tardias (STOLT LROG, et al., 2020; ABREU DROM, et al., 2009)

Foi visto que as informações obtidas em todos os artigos selecionados apenas se agregam, demonstrando pouca divergência entre si, levando em conta os seguintes dados de cada periódico: fatores de risco, perfil epidemiológico analisado e complicações, demonstrados abaixo os dados dispostos no Quadro 1, para título de comparação e exemplificação do que foi dito. 
Quadro 1 - Resultados obtidos após análise dos periódicos.

\begin{tabular}{|c|c|c|c|c|}
\hline Título & Autor / ano de publicação & Fatores de risco & Perfil epidemiológico & Complicações \\
\hline $\begin{array}{l}\text { Morbimortalidade em idosos } \\
\text { por fratura proximal do Fêmur }\end{array}$ & MESQUITA GV, et al., 2009. & $\begin{array}{c}\text { Idade Avançada, Sexo } \\
\text { Feminino e comorbidades } \\
\text { associadas. }\end{array}$ & $\begin{array}{l}4013 \text { idosos que sofreram fratura } \\
\text { proximal do fêmur devido da queda. }\end{array}$ & Osteomielite e morte \\
\hline $\begin{array}{l}\text { Prevalência de quedas em } \\
\text { idosos e fatores Associados }\end{array}$ & SIQUEIRA FV, et al., 2007. & $\begin{array}{c}\text { Idade avançada, sexo } \\
\text { feminino sedentarismo e uso } \\
\text { contínuo de medicações. }\end{array}$ & $\begin{array}{l}4003 \text { idosos assistidos em unidades } \\
\text { básicas de saúde que residem em } 41 \\
\text { municípios de } 7 \text { estados do brasil. }\end{array}$ & Fratura e morte \\
\hline $\begin{array}{l}\text { Internação e mortalidade por } \\
\text { quedas em idosos no brasil: } \\
\text { análise de tendência. }\end{array}$ & ABREU DROM, et al., 2009. & Idade avançada & $\begin{array}{c}\text { Analisados } 66.876 \text { óbitos por quedas } \\
\text { em } 941.923 \text { internações de idosos } \\
\text { mostrados no SIH SUS. }\end{array}$ & Morte \\
\hline $\begin{array}{l}\text { Prevalência de quedas e } \\
\text { fatores associados em idosos }\end{array}$ & CRUZ DT, et al., 2012. & $\begin{array}{l}\text { Idade Avançada e sexo } \\
\text { feminino }\end{array}$ & $\begin{array}{c}420 \text { idosos residentes no município } \\
\text { de Juiz de Fora - MG em um período } \\
\text { de } 12 \text { meses. }\end{array}$ & Fratura \\
\hline $\begin{array}{l}\text { Elevada morbimortalidade e } \\
\text { reduzida taxa de diagnóstico } \\
\text { de osteoporose em idosos } \\
\text { com fratura de fêmur proximal } \\
\text { na cidade de São Paulo. }\end{array}$ & FORTES EM, et al., 2008. & $\begin{array}{l}\text { Idade avançada, sexo } \\
\text { feminino }\end{array}$ & $\begin{array}{l}\text { Analisados } 64 \text { pacientes com } \\
\text { diagnóstico de fratura de fêmur } \\
\text { proximal em dois hospitais escola de } \\
\text { São Paulo - SP. }\end{array}$ & Infecção e morte \\
\hline $\begin{array}{c}\text { Estudo comparativo da } \\
\text { morbimortalidade entre os } \\
\text { idosos no Estado da Paraíba. }\end{array}$ & LIMA MG, et al., 2016. & - & $\begin{array}{c}\text { Dados de internações hospitalares e } \\
\text { mortalidade coletados no SIH e SIM, } \\
\text { de } 410.140 \text { idosos nos anos de } \\
1996,2000,2004 \text { e } 2009 .\end{array}$ & - \\
\hline $\begin{array}{c}\text { Idosos do município do } \\
\text { Recife, estado de } \\
\text { Pernambuco, Brasil: uma } \\
\text { análise da morbimortalidade } \\
\text { hospital }\end{array}$ & SANTOS JS, et., al 2008. & - & $\begin{array}{c}\text { Dados coletados do SIH sobre } \\
1.501 .010 \text { idosos residentes no } \\
\text { município de Recife- PE em } 2005 .\end{array}$ & - \\
\hline $\begin{array}{l}\text { Risk Factors associated with } \\
\text { mortality in Young and long- } \\
\text { lived older adults in } \\
\text { Florianopolis - SC, Brazil }\end{array}$ & CONFORTIN SC, et al., 2020. & Depressão & $\begin{array}{l}\text { Estudo longitudinal com } 1.702 \text { idosos } \\
\text { residentes na área urbana de } \\
\text { Florianópolis nos anos de } 2009, \\
2010,2013 \text { e } 2014 .\end{array}$ & - \\
\hline
\end{tabular}




\begin{tabular}{|c|c|c|c|c|}
\hline Título & Autor / ano de publicação & Fatores de risco & Perfil epidemiológico & Complicações \\
\hline $\begin{array}{c}\text { Increase in fall-related } \\
\text { hospitalization, mortality, and } \\
\text { lethality among older adults in } \\
\text { Brazil. }\end{array}$ & STOLT LROG, et al., 2020. & - & $\begin{array}{l}\text { Dados coletados no SIH sobre } \\
\text { 1.192.829 internações por quedas no } \\
\text { período de janeiro de } 1998 \text { a } \\
\text { novembro de } 2015 \text {, no Brasil. }\end{array}$ & - \\
\hline $\begin{array}{l}\text { Quedas recorrentes e fatores } \\
\text { de risco em idosos } \\
\text { institucionalizados }\end{array}$ & $\begin{array}{l}\text { FERREIRA LMBM, et al., } \\
\qquad 2017 .\end{array}$ & $\begin{array}{c}\text { Institucionalizados e sintoma } \\
\text { de fadiga }\end{array}$ & $\begin{array}{l}\text { Analisadas } 10 \text { instituições em Natal- } \\
\text { RN com total de } 364 \text { idosos. }\end{array}$ & - \\
\hline $\begin{array}{c}\text { Prevalence of mild } \\
\text { hyponatremia and its } \\
\text { association with falls in older } \\
\text { adults admitted to an } \\
\text { emergency geriatric medicine } \\
\text { unit (the mupa unit). }\end{array}$ & BOYER S, et al., 2019. & $\begin{array}{l}\text { Idade avançada, sexo } \\
\text { feminino, hiponatremia, } \\
\text { efeito de medicação e } \\
\text { sarcopenia }\end{array}$ & $\begin{array}{c}\text { Estudo transversal com } 696 \text { idosos } \\
\text { durante } 4 \text { meses na Unidade MUPA } \\
\text { do Centro Hospitalar Universitário De } \\
\text { Limoges (França). }\end{array}$ & - \\
\hline $\begin{array}{l}\text { Burden and correlates of falls } \\
\text { among rural elders of south } \\
\text { india: mobility and } \\
\text { independent living in elders } \\
\text { study. }\end{array}$ & SHARMA PK, et al., 2017. & $\begin{array}{l}\text { História patológica } \\
\text { pregressa, sexo feminino. }\end{array}$ & $\begin{array}{c}\text { Dados de } 562 \text { homens e mulheres > } \\
60 \text { anos coletados entre fevereiro e } \\
\text { novembro de } 2012 \text { em zonas rurais } \\
\text { do sul da índia. }\end{array}$ & - \\
\hline $\begin{array}{l}\text { Falls Among Elderly and Its } \\
\text { Relation with Their Health } \\
\text { Problems And Surrounding } \\
\text { Environmental Factors In } \\
\text { Riyadh }\end{array}$ & $\begin{array}{l}\text { ALSHAMMARI AS, et al., } \\
2018 .\end{array}$ & $\begin{array}{l}\text { História patológica } \\
\text { pregressa, sexo feminino }\end{array}$ & $\begin{array}{c}\text { Estudo transversal em Riyadh Arábia } \\
\text { Saudita com } 357 \text { idosos entre agosto } \\
\text { de } 2015 \text { e abril de } 2016 \text {. }\end{array}$ & - \\
\hline $\begin{array}{c}\text { Injuries related to falls in } \\
\text { elderly patients at a treatment } \\
\text { center } \\
\text { tertiary care in Beirut, } \\
\text { Lebanon }\end{array}$ & ISMAIL RA, et al., 2020. & $\begin{array}{l}\text { Idade avançada e sexo } \\
\text { feminino }\end{array}$ & $\begin{array}{l}\text { Analisados } 253 \text { idosos que sofreram } \\
\text { quedas e foram atendidos em um } \\
\text { pronto socorro em um centro de } \\
\text { atenção terciaria no Líbano. }\end{array}$ & Lesões Pélvicas \\
\hline $\begin{array}{c}\text { Profile and Outcome of } \\
\text { Patients with Falls at Ground } \\
\text { Level. }\end{array}$ & KAISER S, et al., 2020. & Idade avançada. & $\begin{array}{c}\text { Estudados } 596 \text { idosos em um pronto- } \\
\text { socorro de um hospital terciário no } \\
\text { sul da índia. }\end{array}$ & Fraturas e luxações \\
\hline
\end{tabular}




\begin{tabular}{|c|c|c|c|c|}
\hline Título & Autor / ano de publicação & Fatores de risco & Perfil epidemiológico & Complicações \\
\hline $\begin{array}{l}\text { Incidencia de Caídas En El } \\
\text { Hospital De Larga-Media } \\
\text { Estancia. Factores De Riesgo } \\
\text { Y Estrategias Para La } \\
\text { Prevención }\end{array}$ & SANTANA EC, et al., 2019. & $\begin{array}{l}\text { Idade avançada, uso } \\
\text { contínuo de medicação }\end{array}$ & $\begin{array}{l}\text { Realizamos um estudo prospectivo } \\
\text { com } 315 \text { idosos em um centro de } \\
\text { longa permanência por } 12 \text { meses. }\end{array}$ & 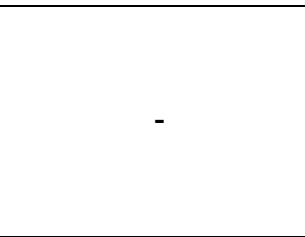 \\
\hline $\begin{array}{l}\text { Prevalence of Risk Factors } \\
\text { For Falls Among Elderly } \\
\text { People Living In Long-Term } \\
\text { Care Homes }\end{array}$ & DHARGAVE P, et al., 2016. & $\begin{array}{l}\text { História Patológica } \\
\text { Pregressa, Sexo Feminino, } \\
\text { Comorbidades Associadas }\end{array}$ & $\begin{array}{l}\text { Estudo transversal com } 163 \text { homens } \\
\text { e mulheres idosos em quatro casas } \\
\text { geriátricas em nagpur, estado de } \\
\text { maharashtra, e bangalore, estado de } \\
\text { karnataka, Índia }\end{array}$ & - \\
\hline $\begin{array}{c}\text { Quedas Em Idosos } \\
\text { Institucionalizados: Riscos, } \\
\text { Consequências e } \\
\text { Antecedentes }\end{array}$ & NETO AHA, et al., 2017. & $\begin{array}{c}\text { Sexo feminino e uso } \\
\text { contínuo de medicamentos }\end{array}$ & $\begin{array}{c}\text { Estudo transversal, realizado com } 45 \\
\text { idosos em instituições de longa } \\
\text { permanência para idosos em João } \\
\text { Pessoa- PB }\end{array}$ & - \\
\hline $\begin{array}{c}\text { Avaliação do risco de quedas } \\
\text { em idosos residentes em } \\
\text { domicílio }\end{array}$ & SMITH AA, et al., 2017. & $\begin{array}{c}\text { Sexo feminino e } \\
\text { comorbidades associadas }\end{array}$ & $\begin{array}{c}\text { Estudo transversal e quantitativo } \\
\text { com } 240 \text { idosos no município de } \\
\text { João Pessoa-PB }\end{array}$ & - \\
\hline $\begin{array}{c}\text { Internação hospitalar, } \\
\text { mortalidade e letalidade } \\
\text { crescente por quedas em } \\
\text { idosos no Brasil }\end{array}$ & STOLT LROG, et al., 2020. & - & $\begin{array}{l}\text { Um estudo descritivo realizado a } \\
\text { partir do SIH-SUS no período de } \\
\qquad 1998 \text { a } 2015\end{array}$ & Fratura \\
\hline $\begin{array}{l}\text { Identificação de fatores } \\
\text { associados a } \\
\text { morbimortalidade de idosos } \\
\text { com fratura de quadril por } \\
\text { fragilidade óssea em } \\
\text { internação hospitalar }\end{array}$ & $\begin{array}{c}\text { MOSQUÉRA JM e ALVES } \\
\text { AR, } 2018 .\end{array}$ & sexo feminino & $\begin{array}{c}\text { Estudo retrospectivo em um serviço } \\
\text { de ortopedia onde foram analisados } \\
347 \text { prontuários de pacientes que } \\
\text { tenham sofrido fratura de quadril por } \\
\text { quedas }\end{array}$ & Fratura e infecção \\
\hline
\end{tabular}

Fonte: Gonçalves MH, et al. 2021. 


\section{DISCUSSÃO}

A Avaliação Geriatria ampla (AGA) é uma ferramenta importante utilizada na área geriátrica com objetivo de avaliar e determinar as deficiências, incapacidades do idoso além de planejar seu cuidado e assistência a médio e longo prazo, tornando assim, essencial para a análise dos fatores intrínsecos e extrínsecos da queda. Os fatores intrínsecos podem ser analisados por meio de exame clínico seguido de uma avaliação neurológica que o principal intuito dessa avaliação é buscar indícios de disfunção cerebelar que possam representa uma alteração do equilíbrio e consequente da sua marcha (MENEZES RL e BACHION MM, 2008; SIQUEIRA FV, 2007).

Além do sistema locomotor, a visão e um importante componente para idoso realizar suas tarefas diárias, sendo assim, patologias como a catarata e a degeneração macular relacionado a idade (DMRI) permite uma diminuição da acuidade visual do idoso o que também pode ocasionar a queda. Idosos frágeis demandam mais atenção pois implicam em desfechos mais desfavoráveis como em uma maior taxa de hospitalização, sofrem mais quedas além da piora nas suas atividades diárias. Sua fisiopatologia e explicada pela redução da atividade de eixos hormonais anabólicos, na instalação da sarcopenia e na presença de um estado inflamatório crônico subliminar (FRIED LP, et al., 2001). Dessa modo, o geriatria precisa analisar as manifestações clinicas porque é um instalação lenta e sempre lembrar dos critérios diagnósticos como redução da força de preensão palmar, redução da velocidade da marcha, perda de peso não intencional, sensação de exaustão e atividade física baixa visto que a presença de três ou mais critérios já fecham o diagnóstico (ABREU DROM, et at., 2018; FRIED LP, et al., 2001).

Outro ponto importante, é avaliação de medicações que o paciente usa de maneira regular, ao pacientes considerado poli farmácia, ou seja, que usam cinco ou mas medicamentos há uma grande chance de os medicamentos como causa etiologia do evento da queda seja por uso inadequado ou por interação medicamentosa. Dessa forma, deve-se tentar otimizar o seu receituário como forma de evitar desfechos desfavoráveis.

Outrossim, é de grande valia verificar o estado nutricional do idoso para a avalição da condição de saúde dele. Essa avaliação é feita com base no Índice de Massa Corporal (IMC), visto que, a obesidade que tem se tornado mais cada vez mais frequente na população geriátrica interfere diretamente no controle postural e no equilíbrio do idoso. Além do mais, em indivíduos obesos, são observadas também alterações na função neuromuscular, na força, na biomecânica, na funcionalidade, na marcha, maior dificuldade frente às desordens externas, maior necessidade de esforço motor e diminuição da independência (MENEZES RL e BACHION MM, 2008; SIQUEIRA FV, 2007; RODRIGUES, et al., 2020)

De igual importância aos itens supracitados, temos o fator hormonal que torna as mulheres mais propensas a quedas e suas complicações. Isto pode ser correlacionado com a queda hormonal, visto na menopausa, lava um desequilíbrio da relação entre os osteoclastos e os osteoblastos que representam respectivamente a formação e quebra da matriz óssea, começando assim, a ocorrer deterioração da microarquitetura óssea o que torna o osso mais frágil, além disso a redução do estrogênio leva a uma diminuição da massa magra e menor força muscular quando comparado ao sexo masculino (MENEZES RL e BACHION MM, 2008; MOSQUÉRA JM e ALVES AR, 2018; SIQUEIRA FV, et al., 2007).

Ainda relacionado a fatores intrínsecos, como visto anteriormente, a osteoporose representa uma peça chave na prevenção das quedas e suas mortalidades, sendo assim o médico ao se deparar com uma paciente do sexo feminino em fase de climatério, é fundamental pensar em fazer um rastreio da osteoporose. Por meio exames complementares como: densitometria óssea e laboratorial: dosagem de cálcio e vitamina $\mathrm{D}$, podemos averiguar essa fragilidade óssea e assim que diagnosticada a osteoporose e instituída a terapia medicamentosa melhores serão resultados e menores os índices de morbidade e mortalidade por quedas (MENEZES RL e BACHION MM, 2008; RIERA R, et al., 2003).

Com relação a história patológica pregressa do paciente, a presença de fraturas prévias já denota que o idoso possui algum grau fragilidade óssea e essa vulnerabilidade aumenta as chances de ocorrência de novas fraturas caso ocorra o evento da queda. Dessa forma, os fatores de risco citados previamente demonstram 
que o fenômeno da queda representa uma alta taxa de fraturas ósseas. Invariavelmente, o tratamento das fraturas é cirúrgico, mas por vezes a queda pode não cominar em fratura, ela apenas aumentam a fragilidade óssea, o que acaba requerendo também alguém tipo de intervenção operatória (STOLT LROG, et al., 2020).

Além dos estudos levantado concordarem sobre a intervenção cirúrgica como terapêutica, eles também mostrarem que metade dos idosos que passaram por um episódio de queda seguida de fratura acabam com incapacidade funcional permanente, incapacidade estas que podem ser desde uma perda da confiança e medo de ocorrência outras quedas, e até mesmo dor crônica, ou limitação de movimentos com restrição da atividade física, que invariavelmente acarretam em um declínio funcional com redução da autonomia (RIERA R, et al., 2003)

No que tange a fatores extrínsecos sabe-se que estes são fortemente influenciadas por condições ambientais como iluminação inadequada, tapetes soltos e superfícies escorregadias. Dessa maneira, avaliação do ambiente domiciliar para identificar e eliminar riscos ambientais tem grande valia como estratégia de prevenção a quedas (RIERA R, et al., 2003). Assuntos como estes precisam abordados na consulta, para que 0 idoso e seus familiares possam saber se prevenir, por conseguinte, se o paciente já tiver alguma limitação física ou alguma necessidade especial o ambiente também deverá ser adaptado de acordo com suas necessidades e seus recursos, para que este tenha ao máximo a sua autonomia preservada.

Não obstante, outro importante aspecto que ainda deve ser abordado pelo médico é a avaliação psicológica desse paciente. A "síndrome pós queda" já é reconhecido hoje como uma sequela ao trauma da queda; se trata da perda da confiança do idoso em sofrer novas quedas ou os agravos em consequentes dela, e mesmo que a síndrome pós queda não tenha aspecto físico expressivo, esta não deve ser negligenciada, pois o idoso acaba perdendo sua independência o que tende a levar uma privação das suas atividades diária, e com isso o isolamento e até mesmo a depressão. Dessa maneira, não podemos omitir o aspecto psicológico da consulta, e se for preciso, a fim de preservar e reforçar sempre a manutenção da autonomia do paciente idoso este pode ser referenciado a equipes multidisciplinares de psicologia, fisioterapia e assistência social (RIERA R, et al., 2003; STOLT LROG, et al., 2020).

\section{CONSIDERAÇÕES FINAIS}

Por fim, pode se inferir que, é essencial realizar um atendimento multidimensional do paciente geriátrico, devemos ser capazes de estimular a manutenção da sua autonomia e independência para a realização das atividades diárias, e também abordar sobre os fatores de risco para quedas buscando sempre interver de maneira apropriada. Mesmo a prevenção sendo o melhor caminho, por vezes o paciente já chega para nós após ocorrido o evento da queda, sendo assim, devemos atuar de forma a reestabelecer sua independência e prevenir maiores morbidades, objetivando a todo momento conservar a qualidade de vida.

\section{REFERÊNCIAS}

1. ABREU DROM, et at. Internação e mortalidade por quedas em idosos no Brasil: análise de tendência. Revista Ciência \& Saúde Coletiva, 2018; 23(4):1131-41.

2. ALSHAMMARI SA, et al. Falls among elderly and its relation with their health problems and surrounding environmental factors in Riyadh. Jounal Family Community Medicine, 2018;25(1): 29-34.

3. BOYER S, et al. Prevalence of mild hyponatremia and its association with falls in older adults admitted to an emergency geriatric medicine unit (the mupa unit). BMC geriatrics, 2019; 19:265

4. CONFORTIN SC, et at. Factors associated with mortality in young and long-lived older adults in Florianopolis, SC, Brazil. Revista Ciência \& Saúde Coletiva,2020 25(6): 2031-40.

5. CRUZ DT, et at. Prevalência de quedas e fatores associados em idosos. Revista Saúde Pública, 2012; 46(1): 13846.

6. DHARGAVE P, et al. Prevalence of Risk Factors for fall among Elderly People Living in Long-Term Care Homes. Journal of Clinical Gerontology \& Geriatrics, 2016: 99-103

7. FERREIRA LMBM, et al. Quedas recorrentes em idosos institucionalizados. Revista Ciência \& Saúde, 2019; 24(1):6775.

8. FORTES EM, et at. Elevada morbimortalidade e reduzida taxa de diagnóstico de osteoporose em idosos com fratura de fêmur proximal na cidade de São Paulo. Arquivo Brasileiro Endocrinologia Metabologia, 2008;52(7): 1106-14. 
9. FRIED LP, et al. Frailty in Older Adults: Evidence for a Phenotype. Journal of Gerontolog. 2001; 56

10. ISMAIL RA, et al. Injuries related to falls in elderly patients at a treatment center tertiary care in Beirut, Lebanon. Journal Emerg Trauma Shock, 2020; 13 (2): 142-145

11. KAISER S, et al. Profile and outcome of patients with falls at ground level. J Family Med Prim Care, 2020; 9 (2): $614-$ 618

12. LIMA MG, et at. Estudo comparativo da morbimortalidade entre idosos no Estado da Paraíba. Revista Brasileira de Educação e Saúde, 2016;6(4): 10-21.

13. MENEZES RL E BACHION MM. Estudo da presença de fatores de riscos intrínsecos para quedas, em idosos institucionalizados. Revista Ciência \& Saúde Coletiva, 2008; 13(4):1209-18.

14. MESQUITA GV, et al. Morbimortalidade Em Idosos Por Fratura Proximal Do Fêmur. Revista Texto \& Contexto Enfermagem, 2009; 18(1): 67-73.

15. MOSQUÉRA JM. Identificação de fatores associados a morbimortalidade de idosos com fraturas de quadril por fragilidade óssea em internação hospitalar. Relatório final de pesquisa de iniciação científica - Centro universitário de Brasilia, 2018; 18p

16. NETO AHA, et al. Quedas em idosos institucionalizados: riscos, consequências e antecedentes. Revista Brasileira de Enfermagem. 2017; 70(4): 719-25

17. RIERA R, et at. Osteoporose - a importância da prevenção de quedas. Revista Brasileira Reumatologia, 2003;43(6): 364-68.

18. RODRIGUES AEC, et al. Mulheres idosas obesas apresentam maior prevalência de quedas e pior equilíbrio estático e dinâmico? Um estudo transversal. 20206 (11): 89242- 54

19. SANTANA EC, et al. Incidencia De Caídas En El Hospital De Larga-Media Estancia. Factores De Riesgo Y Estrategias Para La Prevención. Sociedad Española de neurologia, 2019; (6)

20. SANTOS JS E BARROS MDA. Idosos do Município do Recife, Estado de Pernambuco, Brasil: uma análise da morbimortalidade hospitalar. Epidemiologia e serviços de Saúde, 2008;17(3):177-86.

21. SHARMA PK, et al. Burden and correlates of falls among rural elders of south India: mobility and independent living in elders study. Current Gerontology and Geriatrics Research, 2017; 8

22. SIQUEIRA FV, et al. Prevalência de quedas em idosos e fatores associados. Revista de Saúde Pública, 2007;41(5): 749-56.

23. SMITH AA, et al. Avaliação do risco de quedas em idosos residentes em domicílio. Revista Latino Americana de enfermagem. 2017; 25:e2754

24. STOLT LROG, et al. Increase in fall-related hospitalization, mortality, and lethality among older adults in Brazil. Revista Saúde Pública; 54(76).

25. VIEIRA LS, et al. Quedas em idosos no sul do brasil: prevalências e determinantes. Revista de Saúde Pública, 2018; 55(22). 